\title{
THE INITIAL VALUE PROBLEM FOR MAXWELL'S EQUATIONS FOR TWO MEDIA SEPARATED BY A PLANE ${ }^{1}$
}

\author{
Dedicated to Hans Rademacher \\ on the occasion of his seventieth birthday \\ BY \\ Fritz JoHN
}

Let the column vectors

$$
E=\left(\begin{array}{c}
E_{1} \\
E_{2} \\
E_{3}
\end{array}\right), \quad H=\left(\begin{array}{c}
H_{1} \\
H_{2} \\
H_{3}
\end{array}\right)
$$

describe an electromagnetic field. Denoting the space coordinates by $x_{1}, x_{2}, x_{3}$ and the time by $t$ we put

$$
\xi_{i}=\partial / \partial x_{i}, \quad \tau=\partial / \partial t .
$$

The "curl" operator is then represented by the matrix

$$
C(\xi)=\left(\begin{array}{ccc}
0 & -\xi_{3} & \xi_{2} \\
\xi_{3} & 0 & -\xi_{1} \\
-\xi_{2} & \xi_{1} & 0
\end{array}\right),
$$

while the "divergence" operator corresponds to the row vector

$$
\xi=\left(\xi_{1}, \xi_{2}, \xi_{3}\right) \text {. }
$$

Maxwell's equations for a homogeneous, isotropic, nonconducting medium in the absence of charges then take the form

$$
\varepsilon \tau E=C(\xi) H, \quad \mu \tau H=-C(\xi) E, \quad \xi E=\xi H=0 .
$$

We consider now the case of two media separated by the plane $x_{1}=0$. The field in the medium $x_{1}<0$, where the electric capacities shall have values $\varepsilon, \mu$, we denote by $E, H$. We require that

(1a) $\varepsilon \tau E=C(\xi) H, \quad \mu \tau H=-C(\xi) E, \quad \xi E=\xi H=0$ for $x_{1} \leqq 0$.

The field in the other medium, where the capacities shall have values $\varepsilon^{\prime}, \mu^{\prime}$, we denote by $E^{\prime}, H^{\prime}$. For our purposes it is convenient to use in the second field a new name $x_{1}^{\prime}$ for the first space coordinate $x_{1}$. Putting

$$
\xi_{1}^{\prime}=\partial / \partial x_{1}^{\prime}, \quad \xi^{\prime}=\left(\xi_{1}^{\prime}, \xi_{2}, \xi_{3}\right)
$$

Received April 28, 1961.

1 This paper represents results obtained at the Institute of Mathematical Sciences, New York University, sponsored by the Office of Naval Research, United States Navy. 
we have the equations

$$
\begin{array}{r}
\varepsilon^{\prime} \tau E^{\prime}=C\left(\xi^{\prime}\right) H^{\prime}, \quad \mu^{\prime} \tau H^{\prime}=-C\left(\xi^{\prime}\right) E^{\prime}, \quad \xi^{\prime} E^{\prime}=\xi^{\prime} H^{\prime}=0 \\
\text { for } x_{1}^{\prime} \geqq 0 .
\end{array}
$$

In order to formulate the transition conditions on the interface we introduce the constants

and the matrices

$$
p=\varepsilon / \varepsilon^{\prime}, \quad q=\mu / \mu^{\prime},
$$

$$
P=\left(\begin{array}{ccc}
p & 0 & 0 \\
0 & 1 & 0 \\
0 & 0 & 1
\end{array}\right), \quad Q=\left(\begin{array}{lll}
q & 0 & 0 \\
0 & 1 & 0 \\
0 & 0 & 1
\end{array}\right)
$$

It is then required that

$$
E=P^{-1} E^{\prime}, \quad H=Q^{-1} H^{\prime} \quad \text { for } x_{1}=x_{1}^{\prime}=0 .
$$

Finally the initial values of the field are prescribed:

$$
\begin{aligned}
& E=E^{0}, \quad H=H^{0} \\
& \text { for } x_{1} \leqq 0, \quad t=0 \\
& E^{\prime}=E^{\prime 0}, \quad H^{\prime}=H^{\prime 0} \\
& \text { for } x_{1}^{\prime} \geqq 0, \quad t=0 .
\end{aligned}
$$

The problem to be solved consists in determining a solution of (1a, b, c, d, e) for given initial data $E^{0}, H^{0},{E^{\prime}}^{0}, H^{\prime 0}$. To insure consistency of the data we assume that $E^{0}, H^{0},{E^{\prime}}^{0}, H^{\prime 0}$ are of class $C_{\infty}$ in their respective half-spaces, satisfy

$$
\xi E^{0}=\xi H^{0}=\xi^{\prime} E^{\prime 0}=\xi^{\prime} H^{\prime 0}=0,
$$

and vanish identically near the boundary of those half-spaces.

The transient field corresponding to a pulse in one of the media has been determined by $\mathrm{H}$. Poritsky [7]. With varying degrees of generality this problem has also been discussed by Sommerfeld [8], Gerjuoy [1], van der Pol [10], Weyl [9], and Garnir [2]. The method used in the present paper is based on a principle used previously by the author to solve the corresponding acoustical problem. (See [3].) It furnishes the solution in terms of finite quadratures extended over the initial values without intervention of any Fourier and Laplace transforms. In fact the problem is reduced to that of solving the boundary-initial value problem for the ordinary wave equation in a half-space, which has a well known simple explicit solution. (See Freda [6].) The resulting solution of the two-media problem obtained here lends itself to the determination of the domains of dependence, which are found to agree with those suggested by geometrical optics.

\section{The principle}

Given a field $E^{\prime}, H^{\prime}$ for $x_{1}^{\prime} \geqq 0$ and all $t$ satisfying Maxwell's equations (1b), there cannot exist more than one "complementary" field $E, H$ for $x_{1} \leqq 0$ 
satisfying Maxwell's equations (1a) and the transition conditions (1c). Indeed (1a), (1c) constitute a Cauchy problem (though an improperly posed one), whose solution is determined uniquely by a well known theorem of Holmgren [5] (see also John [4]). There does not always exist a complementary field. Take, for example, a time-independent field

$$
E^{\prime}=\operatorname{grad} \phi\left(x_{1}^{\prime}, x_{2}, x_{3}\right), \quad H^{\prime}=0,
$$

where $\phi$ is harmonic for $x_{1}^{\prime} \geqq 0$. Then there will not exist a complementary field, unless $\phi$ can be continued as a harmonic function into the whole $x_{1}^{\prime} x_{2} x_{3}$ space. We call a field $E^{\prime}, H^{\prime}$ compatible, if a complementary field exists. We have then the following principle:

$$
\text { If }
$$

$$
p q=\varepsilon \mu / \varepsilon^{\prime} \mu^{\prime}>1
$$

(i.e., the right-hand medium has the larger propagation speed), and if $E^{\prime}\left(x_{1}^{\prime}, x_{2}, x_{3}, t\right), H^{\prime}\left(x_{1}^{\prime}, x_{2}, x_{3}\right)$ form a compatible field, then the complementary field $E, H$ can be extended into the whole $x_{1} x_{2} x_{3}$-space. Moreover with $E^{\prime}, H^{\prime}$ also the translated vectors $E^{\prime}\left(x_{1}^{\prime}+y, x_{2}, x_{3}, t\right)$, $H^{\prime}\left(x_{1}^{\prime}+y, x_{2}, x_{3}, t\right)$ form a compatible field for any positive constant $y$.

We shall see how this principle leads to the construction of the solution of the initial value problem ( $1 \mathrm{a}, \mathrm{b}, \mathrm{c}, \mathrm{d}, \mathrm{e})$ and will itself be verified by that construction. Assume we had a solution of that problem. The principle asserts that there exist functions $e\left(x_{1}, y, x_{2}, x_{3}, t\right), h\left(x_{1}, y, x_{2}, x_{3}, t\right)$ for $y \geqq 0$ satisfying

$$
\varepsilon \tau e=C(\xi) h, \quad \mu \tau h=-C(\xi) e, \quad \xi e=\xi h=0
$$

for which

$$
\begin{aligned}
e\left(0, y, x_{2}, x_{3}, t\right) & =P^{-1} E^{\prime}\left(y, x_{2}, x_{3}, t\right), \\
h\left(0, y, x_{2}, x_{3}, t\right) & =Q^{-1} H^{\prime}\left(y, x_{2}, x_{3}, t\right) .
\end{aligned}
$$

We write again $x_{1}^{\prime}$ for $y$, and consider $e, h$ as functions of the independent variables $x_{1}, x_{1}^{\prime}, x_{2}, x_{3}, t$, which satisfy (4) for $x_{1}^{\prime} \geqq 0$ and also

$$
\begin{array}{lll}
e=P^{-1} E^{\prime}, & h=Q^{-1} H^{\prime} & \text { for } x_{1}=0, \quad x_{1}^{\prime} \geqq 0, \\
e=E, & h=H & \text { for } x_{1}^{\prime}=0, \quad x_{1} \leqq 0 .
\end{array}
$$

Equations (4) imply that

$$
L e=L h=0 \quad \text { for } x_{1} \leqq 0, \quad x_{1}^{\prime} \geqq 0,
$$

where $L$ is the scalar wave operator

$$
L=\tau^{2}-(1 / \varepsilon \mu)\left(\xi_{1}^{2}+\xi_{2}^{2}+\xi_{3}^{2}\right) .
$$


From (5), (4), (1b) we get for $x_{1}=0, x_{1}^{\prime} \geqq 0$

$$
\begin{gathered}
0=\varepsilon \tau\left(e-P^{-1} E^{\prime}\right)=C(\xi) h-p P^{-1} C\left(\xi^{\prime}\right) H^{\prime}=\left(C(\xi)-p P^{-1} C\left(\xi^{\prime}\right) Q\right) h, \\
0=\xi h-\xi^{\prime} H^{\prime}=\left(\xi-\xi^{\prime} Q\right) h .
\end{gathered}
$$

If we introduce the vectors

$$
u=\left(C(\xi)-p P^{-1} C\left(\xi^{\prime}\right) Q\right) h, \quad v=\left(C(\xi)-q Q^{-1} C\left(\xi^{\prime}\right) P\right) e
$$

and the scalars

$$
\phi=\left(\xi-\xi^{\prime} Q\right) h, \quad \psi=\left(\xi-\xi^{\prime} P\right) e,
$$

we have

$$
u=v=\phi=\psi=0 \quad \text { for } x_{1}=0, \quad x_{1}^{\prime} \geqq 0 .
$$

Writing relations $(7 \mathrm{a}, \mathrm{b})$ componentwise we observe that $u_{1}$ and $v_{1}$ vanish identically, and that $(7 \mathrm{a}, \mathrm{b})$ are equivalent to the relations

$$
\begin{aligned}
& \xi_{1} h_{1}=q \xi_{1}^{\prime} h_{1}+\phi, \\
& \xi_{1} h_{2}=p \xi_{1}^{\prime} h_{2}-(p q-1) \xi_{2} h_{1}+u_{3}, \\
& \xi_{1} h_{3}=p \xi_{1}^{\prime} h_{3}-(p q-1) \xi_{3} h_{1}-u_{2}, \\
& \xi_{1} e_{1}=p \xi_{1}^{\prime} e_{1}+\psi, \\
& \xi_{1} e_{2}=q \xi_{1}^{\prime} e_{2}-(p q-1) \xi_{2} e_{1}+v_{3}, \\
& \xi_{1} e_{3}=q \xi_{1}^{\prime} e_{3}-(p q-1) \xi_{3} e_{1}-v_{2} .
\end{aligned}
$$

It is clear that also

$$
L u=L v=L \phi=L \psi=0 \quad \text { for } x_{1}^{\prime} \geqq 0 .
$$

Since $u, v, \phi, \psi$ vanish on $x_{1}=0$ and the operator $L$ is even in $\xi_{1}$, it follows that $u, v, \phi, \psi$ must be odd functions of $x_{1}$. We form now the operator

$$
L^{\prime}=\tau^{2}-\left(1 / \varepsilon^{\prime} \mu^{\prime}\right)\left(\xi_{1}^{\prime 2}+\xi_{2}^{2}+\xi_{3}^{2}\right) .
$$

By (1b) and (5)

$$
L^{\prime} e=L^{\prime} h=0 \quad \text { for } x_{1}=0, \quad x_{1}^{\prime} \geqq 0 .
$$

It follows from relations $(8 \mathrm{a}, \mathrm{b}, \mathrm{c}, \mathrm{d}, \mathrm{e}, \mathrm{f})$ and $(7 \mathrm{c})$ that then also

$$
\xi_{1} L^{\prime} e=\xi_{1} L^{\prime} h=0 \quad \text { for } x_{1}=0, \quad x_{1}^{\prime} \geqq 0 .
$$

$L^{\prime} e$ and $L^{\prime} h$ are then as functions of $x_{1}, x_{2}, x_{3}, t$ solutions of the wave equation

$$
L\left(L^{\prime} e\right)=0, \quad L\left(L^{\prime} h\right)=0 \quad \text { for } x_{1} \leqq 0
$$

with vanishing Cauchy data on the plane $x_{1}=0$, and hence vanish identically:

$$
L^{\prime} e=L^{\prime} h=0 \quad \text { for } x_{1}^{\prime} \geqq 0 .
$$


We finally introduce the operator

$$
\Lambda=\varepsilon \mu\left(L^{\prime}-L\right)=\xi_{1}^{2}-p q \xi_{1}^{\prime 2}-(p q-1)\left(\xi_{2}^{2}+\xi_{3}^{2}\right) .
$$

By (6), (10), $e$ and $h$ satisfy the time-independent differential equations

$$
\Lambda e=\Lambda h=0 \quad \text { for } x_{1}^{\prime} \geqq 0 .
$$

The important point is that $\Lambda$ is a hyperbolic operator by assumption (3) with $x_{1}$ as time-like direction.

The construction of the solution of our problem starts now with the determination of the initial values $e^{0}, h^{0}$ of $e, h$ for $t=0$. One first obtains $e^{0}, h^{0}$ for $x_{1}^{\prime} \geqq 0, x_{1} \leqq 0$ as the solution of the boundary-initial value problem

$$
\begin{array}{cc}
\Lambda e^{0}=\Lambda h^{0}=0 & \text { for } x_{1} \leqq 0, \quad x_{1}^{\prime} \geqq 0, \\
e^{0}=E^{0}, \quad h^{0}=H^{0} & \text { for } x_{1} \leqq 0, \quad x_{1}^{\prime}=0, \\
e^{0}=P^{-1}{E^{\prime}}^{0}, \quad h^{0}=Q^{-1} H^{\prime 0}, & \\
0=\left(C(\xi)-p P^{-1} C\left(\xi^{\prime}\right) Q\right) h^{0}=\left(C(\xi)-q Q^{-1} C\left(\xi^{\prime}\right) P\right) e^{0} & \\
=\left(\xi-\xi^{\prime} Q\right) h^{0}=\left(\xi-\xi^{\prime} P\right) e^{0} & \text { for } x_{1}=0, \quad x_{1}^{\prime} \geqq 0 .
\end{array}
$$

(By (8a, b, c, d, e, f) relations (13c) amount to prescribing the Cauchy data of $e, h$ on $x_{1}=0, x_{1}^{\prime} \geqq 0$.) With the $e^{0}, h^{0}$ determined we form the functions

$$
\begin{aligned}
& u^{0}=\left(C(\xi)-p P^{-1} C\left(\xi^{\prime}\right) Q\right) h^{0}, \quad v^{0}=\left(C(\xi)-q Q^{-1} C\left(\xi^{\prime}\right) P\right) e^{0}, \\
& \text { (13e) } \phi^{0}=\left(\xi-\xi^{\prime} Q\right) h^{0} \text {, } \\
& \psi^{0}=\left(\xi-\xi^{\prime} P\right) e^{0}
\end{aligned}
$$

for $x_{1} \leqq 0, x_{1}^{\prime} \geqq 0$. We extend these functions into the region $x_{1} \geqq 0, x_{1}^{\prime} \geqq 0$ as odd functions of $x_{1}$. We then determine $e^{0}, h^{0}$ in the region $x_{1} \geqq 0, x_{1}^{\prime} \geqq 0$ from the relations $\left(13 \mathrm{~d}\right.$, e) using the extended functions $u^{0}, v^{0}, \phi^{0}, \psi^{0}$, and requiring that $e^{0}=P^{-1}{E^{\prime}}^{0}, h^{0}=Q^{-1} H^{\prime 0}$ on $x_{1}=0, x_{1}^{\prime} \geqq 0$. In this way we obtain $e^{0}, h^{0}$ for $x_{1}^{\prime} \geqq 0$ and all $x_{1}, x_{2}, x_{3}$. The vectors $e, h$ can then be obtained for $x_{1}^{\prime} \geqq 0$ and all $x_{1}, x_{2}, x_{3}, t$ as solutions of the pure initial value problem

$$
\begin{aligned}
& L e=L h=0, \\
& e=e^{0}, \quad h=h^{0}, \quad \varepsilon \tau e=C(\xi) h^{0}, \quad \mu \tau h=-C(\xi) e^{0} \quad \text { for } t=0 .
\end{aligned}
$$

Finally the desired field vectors $E, H, E^{\prime}, H^{\prime}$ are given by

$$
\begin{array}{rlrl}
E & =e\left(x_{1}, 0, x_{2}, x_{3}, t\right), & H & =h\left(x_{1}, 0, x_{2}, x_{3}, t\right), \\
E^{\prime} & =P e\left(0, x_{1}^{\prime}, x_{2}, x_{3}, t\right), \quad H^{\prime}=Q h\left(0, x_{1}^{\prime}, x_{2}, x_{3}, t\right) \quad \text { for } x_{1}^{\prime} \geqq 0 .
\end{array}
$$

\section{Verification of the solution}

The first step in the construction consists in the solution of the Cauchy problem $(13 \mathrm{a}, \mathrm{b}, \mathrm{c})$ for $e^{0}, h^{0}$ in the quarter-space $x_{1} \leqq 0, x_{1}^{\prime} \geqq 0$. Under the assumptions made that the data are in $C_{\infty}$ and vanish near the intersection of the planes $x_{1}=0, x_{1}^{\prime}=0$, a unique solution $e^{0}, h^{0}$ of class $C_{\infty}$ will exist. 
The solution can be written conveniently by associating with the differential operator $\Lambda$ the metric represented by the quadratic form

$$
R(y)=R\left(y_{1}, y_{1}^{\prime}, y_{2}, y_{3}\right)=y_{1}^{2}-\frac{1}{p q} y_{1}^{\prime 2}-\frac{1}{p q-1}\left(y_{2}^{2}+y_{3}^{2}\right) .
$$

The solution $e^{0}$ consists of a contribution of the initial values of $e^{0}, \xi_{1} e^{0}$ for $x_{1}=0, x_{1}^{\prime} \geqq 0$ and of a contribution of the boundary values of $e^{0}$ for $x_{1}^{\prime}=0$, $x_{1} \leqq 0$. The contribution of the initial values is identical with the solution of the pure initial value problem obtained by extending the initial values of $e^{0}, \xi_{1} e^{0}$ for $x_{1}=0$ to all $x_{1}^{\prime}, x_{2}, x_{3}$ as odd functions of $x_{1}^{\prime}$. The contribution to $e^{0}$ at a point $x=\left(x_{1}, x_{1}^{\prime}, x_{2}, x_{3}\right)$ of the initial data is then given by the formula

$$
\frac{1}{4 \pi} \frac{\partial}{\partial x_{1}} \frac{1}{x_{1}} \iint_{\substack{R(y-x)=0 \\ y_{1}=0}} e^{0}(y) d \omega+\frac{1}{4 \pi} \frac{1}{x_{1}} \iint_{\substack{R(y-x)=0 \\ y_{1}=0}} \xi_{1} e^{0}(y) d \omega .
$$

The contribution of the boundary data is given by

$$
-\frac{p q}{2 \pi} \frac{\partial}{\partial x_{1}^{\prime}} \frac{1}{x_{1}^{\prime}} \iint_{\substack{R(y-x)=0 \\ y_{1}^{\prime}=0 \\ x_{1}<y_{1}<0}} e^{0}(y) d \omega
$$

Here in each case $d \omega$ represents the surface element induced by the metric $R$, i.e., in (15a)

$$
d \omega=\left|\frac{\sqrt{p q} x_{1} d y_{2} d y_{3}}{(p q-1)\left(x_{1}^{\prime}-y_{1}^{\prime}\right)}\right|
$$

while in $(15 b)$

$$
d \omega=\left|\frac{x_{1}^{\prime} d y_{2} d y_{3}}{\sqrt{p q}(p q-1)\left(x_{1}-y_{1}\right)}\right| .
$$

Defining next $u^{0}, v^{0}, \phi^{0}, \psi^{0}$ by (13d, e) we have that those expressions are in $C_{\infty}$ for $x_{1} \leqq 0, x_{1}^{\prime} \geqq 0$, satisfy

$$
\Lambda u^{0}=\Lambda v^{0}=\Lambda \phi^{0}=\Lambda \psi^{0}=0,
$$

and vanish for $x_{1}=0, x_{1}^{\prime} \geqq 0$. By continuing them as odd functions of $x_{1}$ into $x_{1} \geqq 0, x_{1}^{\prime} \geqq 0$, they stay solutions of (16a), and the extended functions are of class $C_{\infty}$ for $x_{1}^{\prime} \geqq 0$.

The functions $e^{0}, h^{0}$ are obtained for $x_{1} \geqq 0, x_{1}^{\prime} \geqq 0$ by means of equations (13d, e), using initial conditions $e^{0}=P^{-1} E^{\prime}, h^{0}=Q^{-1} H^{\prime 0}$ for $x_{1}=0, x_{1}^{\prime} \geqq 0$. Writing (13d) in the equivalent form (8a, b, c, d, e, f) with superscripts 0 added, we see that $e_{1}^{0}, e_{2}^{0}, e_{3}^{0}, h_{1}^{0}, h_{2}^{0}, h_{3}^{0}$ can be obtained successively by quadratures along the lines

$p d x_{1}+d x_{1}^{\prime}=d x_{2}=d x_{3}=0$ respectively $q d x_{1}+d x_{1}^{\prime}=d x_{2}=d x_{3}=0$. 
Since $p$ and $q$ are positive, these lines issuing from points with $x_{1}=0, x_{1}^{\prime} \geqq 0$ cover the whole quarter-space $x_{1} \geqq 0, x_{1}^{\prime} \geqq 0$. The resulting extended functions $e^{0}, h^{0}$ are again in $C_{\infty}$ for all $x_{1}^{\prime} \geqq 0$ and all $x_{1}, x_{2}, x_{3}$. Moreover they again satisfy (13a), since $\Lambda e^{0}$ and $\Lambda h^{0}$ vanish for $x_{1}=0$, and in addition satisfy first order equations obtained from (13d, e) by applying the $\Lambda$-operator, with the inhomogeneous parts vanishing by (16a).

We now make use of the identity

$$
\begin{aligned}
\left(C(\xi)-p P^{-1} C\left(\xi^{\prime}\right) Q\right) C(\xi) & +p P^{-1} C\left(\xi^{\prime}\right) Q\left(C(\xi)-q Q^{-1} C\left(\xi^{\prime}\right) P\right) \\
& =C^{2}(\xi)-p q P^{-1} C^{2}\left(\xi^{\prime}\right) P \\
& =\left(\xi^{T}-p q P^{-1} \xi^{\prime T}\right) \xi+p q P^{-1} \xi^{\prime T}\left(\xi-\xi^{\prime} P\right)-\Lambda I,
\end{aligned}
$$

where $I$ denotes the unit-matrix, and $\xi^{T}, \xi^{\prime T}$ are the transposed vectors to $\xi$ and $\xi^{\prime}$, i.e., the gradient operators. Applying this identity to $e^{0}$, it becomes

$$
\begin{aligned}
\left(C(\xi)-p P^{-1} C\left(\xi^{\prime}\right) Q\right) C(\xi) e^{0}+p P^{-1} C\left(\xi^{\prime}\right) Q v^{0} \\
=\left(\xi^{T}-p q P^{-1} \xi^{\prime T}\right) \xi e^{0}+p q P^{-1} \xi^{\prime T} \psi^{0} .
\end{aligned}
$$

The first row of this identity reads

$$
-\xi_{3} v_{2}^{0}+\xi_{2} v_{3}^{0}=\left(\xi_{1}-q \xi_{1}^{\prime}\right) \xi e^{0}+q \xi_{1}^{\prime} \psi^{0} .
$$

By $(13 \mathrm{c})$, (2) we have for $x_{1}=0, x_{1}^{\prime} \geqq 0$

$$
\xi e^{0}=\xi^{\prime} P e^{0}+\psi^{0}=\xi^{\prime} E^{\prime 0}=0 .
$$

Then also by (16c)

$$
\xi_{1} \xi e^{0}=q \xi_{1}^{\prime} \xi e^{0}=0 \quad \text { for } x_{1}=0, \quad x_{1}^{\prime} \geqq 0,
$$

since $v^{0}$ and $\psi^{0}$ vanish there. Moreover by (13b), (2)

$$
\xi e^{0}=\xi E^{0}=0 \quad \text { for } x_{1}^{\prime}=0, \quad x_{1} \leqq 0 .
$$

Then $\xi e^{0}$ is a solution of the differential equation

$$
\Lambda \xi e^{0}=0 \quad \text { for } x_{1} \leqq 0, \quad x_{1}^{\prime} \geqq 0
$$

with vanishing boundary and initial data. It follows that $\xi e^{0}$ vanishes in the quarter-space $x_{1} \leqq 0, x_{1}^{\prime} \geqq 0$. Then also $\left(\xi_{1}-q \xi_{1}^{\prime}\right) \xi e^{0}$ vanishes there. By (16c) this expression is an odd function of $x_{1}$, since $v^{0}$ and $\psi^{0}$ are odd. Hence also

$$
\left(\xi_{1}-q \xi_{1}^{\prime}\right) \xi e^{0}=0 \quad \text { for } x_{1} \geqq 0, \quad x_{1}^{\prime} \geqq 0 .
$$

Since $\xi e^{0}$ vanishes on $x_{1}=0, x_{1}^{\prime} \geqq 0$, this implies that $\xi e^{0}=0$ also for $x_{1} \geqq 0$, $x_{1}^{\prime} \geqq 0$. Similarly for $\xi h^{0}$. We have then

$$
\xi e^{0}=\xi h^{0}=0 \quad \text { for } x_{1}^{\prime} \geqq 0 .
$$

Identity $(16 \mathrm{~b})$ then yields that

$$
\left(C(\xi)-p P^{-1} C\left(\xi^{\prime}\right) Q\right) C(\xi) e^{0}
$$


and similarly

$$
\left(C(\xi)-q Q^{-1} C\left(\xi^{\prime}\right) P\right) C(\xi) h^{0}
$$

are odd in $x_{1}$ for $x_{1}^{\prime} \geqq 0$.

The functions $e, h$ of $x_{1}, x_{1}^{\prime}, x_{2}, x_{3}, t$ are defined for $x_{1}^{\prime} \geqq 0$ as solutions of the pure initial value problem (13f, g). They can be obtained in terms of $e^{0}, h^{0}$ by an explicit formula of the type (15a), and are again of class $C_{\infty}$. We have for $t=0, x_{1}^{\prime} \geqq 0$

and

$$
\varepsilon \tau e-C(\xi) h=C(\xi) h^{0}-C(\xi) h^{0}=0,
$$

$$
\begin{aligned}
\mu \tau(\varepsilon \tau e-C(\xi) h) & =\varepsilon \mu \tau^{2} e-C(\xi) \mu \tau h \\
& =\varepsilon \mu(L e)_{t=0}+\xi \xi^{T} e^{0}+C^{2}(\xi) e^{0} \\
& =\xi^{T} \xi e^{0}=0
\end{aligned}
$$

as a consequence of $(16 \mathrm{~d})$. Thus $\varepsilon \tau e-C(\xi) h$ is a solution of the hyperbolic equation

$$
L(\varepsilon \tau e-C(\xi) h))=0
$$

with vanishing initial data, and hence vanishes identically. Similarly for $\mu \tau h+C(\xi) e$. Consequently $e$ and $h$ satisfy Maxwell's equations

$$
\varepsilon \tau e=C(\xi) h, \quad \mu \tau h=-C(\xi) e \quad \text { for } x_{1}^{\prime} \geqq 0 .
$$

These equations imply that $\xi e$ and $\xi h$ are independent of $t$, and thus

$$
\xi e=\xi h=0 \quad \text { for } x_{1}^{\prime} \geqq 0,
$$

since (16f) holds for $t=0$ by (16d).

We define $u, v$ for $x_{1}^{\prime} \geqq 0$ by $(7 \mathrm{a})$. All these functions are annihilated by the operator $L$. The initial values for $u$ at $t=0$ are

$$
\begin{gathered}
u=u^{0}, \\
\tau u=\left(C(\xi)-p P^{-1} C\left(\xi^{\prime}\right) Q\right) \tau h=-(1 / \mu)\left(C(\xi)-p P^{-1} C\left(\xi^{\prime}\right) Q\right) C(\xi) e^{0} .
\end{gathered}
$$

Since these initial values have been shown to be odd functions of $x_{1}$, it follows that $u$ is an odd function of $x_{1}$ for all $t$. Similarly $v$ is seen to be odd in $x_{1}$.

The field $E^{\prime}, H^{\prime}$ is defined by (13i). It is clear that $E^{\prime}, H^{\prime}$ are in $C_{\infty}$ for $x_{1}^{\prime} \geqq 0$. We have by $(16 \mathrm{e})$

$$
\begin{aligned}
\mathcal{\varepsilon}^{\prime} \tau E^{\prime}-C\left(\xi^{\prime}\right) H^{\prime} & =\left(\varepsilon^{\prime} \tau P e-C\left(\xi^{\prime}\right) Q h\right)_{x_{1}=0} \\
& =\left(p^{-1} P\left(C(\xi)-p P^{-1} C\left(\xi^{\prime}\right) Q\right) h\right)_{x_{1}=0} \\
& =\left(p^{-1} P u\right)_{x_{1}=0}=0
\end{aligned}
$$

since the odd function $u$ vanishes for $x_{1}=0$. We see then that $E^{\prime}, H^{\prime}$ satisfy Maxwell's equations

$$
\varepsilon^{\prime} \tau E^{\prime}=C\left(\xi^{\prime}\right) H^{\prime}, \quad \mu^{\prime} \tau H^{\prime}=-C\left(\xi^{\prime}\right) E^{\prime} \quad \text { for } x_{1}^{\prime} \geqq 0 .
$$


The remaining equations

$$
\xi^{\prime} E^{\prime}=\xi^{\prime} H^{\prime}=0
$$

follow from $(16 \mathrm{~g})$, if we observe that $E^{\prime}, H^{\prime}$ have for $t=0$ the initial values

$$
E^{\prime}=(P e)_{t=0, x_{1}=0}=\left(P e^{0}\right)_{x_{1}=0}=E^{\prime 0} \quad H^{\prime}=H^{\prime 0},
$$

which satisfy (16h).

Finally the field $E, H$ is defined by (13h) for all arguments $x_{1}, x_{2}, x_{3}, t$. It satisfies Maxwell's equations as the special case $x_{1}^{\prime}=0$ of $(16 \mathrm{e}, \mathrm{f})$. For $t=0, x_{1}^{\prime} \leqq 0$ we have the initial values

$$
E=(e)_{x_{1}^{\prime}=0, t=0}=\left(e^{0}\right)_{x_{1}^{\prime}=0}=E^{0}, \quad H=H^{0} .
$$

The transition conditions

$$
E=P^{-1} E^{\prime}, \quad H=Q^{-1} H^{\prime} \quad \text { for } x_{1}=x_{1}^{\prime}=0
$$

are a direct consequence of the definitions $(13 \mathrm{~h}, \mathrm{i})$ of $E, H, E^{\prime}, H^{\prime}$. This completes the solution of the original problem.

\section{Discussion of the solution}

We first consider the effect of an initial disturbance concentrated at a single point $\left(\eta^{\prime}, 0,0\right)$ of the medium with the larger speed, i.e., $\eta^{\prime}>0$. We first have to determine the domain of influence on $e^{0}, h^{0}$. In the quarter-space $x_{1} \leqq 0, x_{1}^{\prime} \geqq 0$ the vector $e^{0}$ is given by formula (15a), since by assumption the boundary values (13b) of $e^{0}$ vanish. Since the initial data on $x_{1}=0$ are to be continued as odd functions of $x_{1}^{\prime}$, only the point

$$
y=\left(y_{1}, y_{1}^{\prime}, y_{2}, y_{3}\right)=\left(0, \eta^{\prime}, 0,0\right)
$$

and the symmetric point

$$
\bar{y}=\left(y_{1},-y_{1}^{\prime}, y_{2}, y_{3}\right)=\left(0,-\eta^{\prime}, 0,0\right)
$$

make a contribution. It follows that $e^{0}, h^{0}$ have their support in $x_{1} \leqq 0$, $x_{1}^{\prime} \geqq 0$ in the union of the cones

$$
\begin{aligned}
& R(x-y)=x_{1}^{2}-\frac{1}{p q}\left(x_{1}^{\prime}-\eta^{\prime}\right)^{2}-\frac{1}{p q-1}\left(x_{2}^{2}+x_{3}^{2}\right)=0, \\
& R(x-\bar{y})=x_{1}^{2}-\frac{1}{p q}\left(x_{1}^{\prime}+\eta^{\prime}\right)^{2}-\frac{1}{p q-1}\left(x_{3}^{2}+x_{3}^{2}\right)=0 .
\end{aligned}
$$

The expressions $u^{0}, v^{0}, \phi^{0}, \psi^{0}$ then have their support on the same cones. They are continued into $x_{1} \geqq 0, x_{1}^{\prime} \geqq 0$ as odd functions of $x_{1}$ and hence have their support in that quarter-space again on the same cones. It follows that $u^{0}, v^{0}, \phi^{0}, \psi^{0}$ vanish in the set

$$
x_{1}^{2}-\frac{1}{p q}\left(x_{1}^{\prime}-\eta^{\prime}\right)^{2}-\frac{1}{p q-1}\left(x_{2}^{2}+x_{3}^{2}\right)<0, \quad x_{1}^{\prime} \geqq 0, \quad x_{1} \geqq 0 .
$$


The vectors $e^{0}, h^{0}$ are continued into $x_{1} \geqq 0, x_{1}^{\prime} \geqq 0$ by equations (13d, e). We shall show that they also vanish in the set (17).

We write the equations (13d, e) in the form $(8 a, b, c, d, e, f)$. We have e.g.

Using the identity

$$
\left(\xi_{1}-q \xi_{1}^{\prime}\right) h_{1}^{0}=\phi^{0} .
$$

$$
p\left(\xi_{1}-q \xi_{1}^{\prime}\right)^{2}=(p-q) \Lambda+q\left(\xi_{1}-p \xi_{1}^{\prime}\right)^{2}+(p-q)(p q-1)\left(\xi_{2}^{2}+\xi_{3}^{2}\right)
$$

and the fact that $\Lambda h_{1}^{0}=0$, we obtain for $h_{1}^{0}$ the second order equation

$$
T h_{1}^{0}=p\left(\xi_{1}-q \xi_{1}^{\prime}\right) \phi^{0},
$$

where

$$
T=q\left(\xi_{1}-p \xi_{1}^{\prime}\right)^{2}+(p-q)(p q-1)\left(\xi_{2}^{2}+\xi_{3}^{2}\right) .
$$

Since $T$ is a degenerate quadratic form, the differential equation (18) connects the values of $h_{1}^{0}$ and $\phi$ in each hyperplane $p x_{1}+x_{1}^{\prime}=$ const.

Consider now a plane $p x_{1}+x_{1}^{\prime}=$ const. $=c>0$. In that plane we can introduce $x_{1}, x_{2}, x_{3}$ as independent variables. The operator $T$ in those variables takes the form

$$
T=q \frac{\partial^{2}}{\partial x_{1}^{2}}+(p-q)(p q-1)\left(\frac{\partial^{2}}{\partial x_{2}^{2}}+\frac{\partial^{2}}{\partial x_{3}^{2}}\right) .
$$

In that plane $T h_{1}^{0}$ and the initial data of $h_{1}^{0}$ vanish for

$$
\begin{aligned}
& (q-p)\left[\frac{1}{q}\left(x_{1}+\frac{c-\eta^{\prime}}{q-p}\right)^{2}\right. \\
& \left.+\frac{1}{(p-q)(p q-1)}\left(x_{2}^{2}+x_{3}^{2}\right)-\frac{\left(c-\eta^{\prime}\right)^{2}}{p(p-q)^{2}}\right]<0 ; \\
& \quad 0<x_{1}<c / p .
\end{aligned}
$$

For $p>q$ the operator (20) is elliptic, and hence $h_{1}^{0}$ is analytic in the set (20). Moreover that set is connected and contains a neighborhood of the point $x_{1}=x_{2}=x_{3}=0$, where the Cauchy data vanish. It follows that $h_{1}^{0}$ vanishes everywhere in the set (20).

For $p<q$ the operator $T$ is hyperbolic. The set (21) in that case is bounded by the plane $x_{1}=0$, a portion of the plane $x_{1}=c / p$ and a portion of one sheet of a hyperbolic surface. The asymptotic cone of the hyperbolic surface is a characteristic cone with respect to the operator (20). It follows that vanishing Cauchy data on $x_{1}=0$ imply again vanishing of $h_{1}^{0}$ throughout the set (21). Since every point of the set (17) lies in a suitable plane $p x_{1}+x_{1}^{\prime}=c$, it follows that $h_{1}^{0}$ vanishes throughout the set (17).

The same argument yields that $e_{1}^{0}=0$ in (17). Then also the remaining components of $e^{0}, h^{0}$ vanish in (17), since each of them is annihilated either by $\xi_{1}-p \xi_{1}^{\prime}$ or $\xi_{1}-q \xi_{1}^{\prime}$ in the set (17). 
For $x_{1}^{\prime}=0$ we find that the initial values of $E, H$ as solution of the differential equations $L E=L H=0$ have their support in the set

$$
x_{1}^{2}-\frac{1}{p q-1}\left(x_{2}^{2}+x_{3}^{2}\right) \geqq \frac{1}{p q}{\eta^{\prime}}^{2}, \quad x_{1}>0 .
$$

This set consists of the interior of one sheet of a hyperboloid of revolution with focus at the point $\left(\eta^{\prime}, 0,0\right)$, vertex at $\left(\sqrt{\varepsilon^{\prime} \mu^{\prime} / \varepsilon \mu} \eta^{\prime}, 0,0\right)$, and center at the origin. The field $E, H$ is identical with the field obtained in a material with constants $\varepsilon, \mu$ extending throughout the whole space and with a suitable initial disturbance distributed over the set (22).

We introduce the propagation speeds in the two media, which are respectively

$$
c=1 / \sqrt{\varepsilon \mu}, \quad c^{\prime}=1 / \sqrt{\varepsilon^{\prime} \mu^{\prime}} .
$$

At the time $t$ the support of $E, H$ will be in the $c t$-neighborhood of the set (22). This neighborhood will be bounded by the outer parallel surface of distance $c t$ to the hyperbolic surface

$$
x_{1}^{2}-\frac{1}{p q-1}\left(x_{2}^{2}+x_{3}^{2}\right)=\frac{1}{p q}{\eta^{\prime}}^{2}, \quad x_{1}>0 .
$$

The boundary surface of the $c t$-neighborhood of (22), i.e., the wave front at the time $t$, can also be obtained by laying off the distance $c t$ along the outer normal of any point of (23). Only that portion of the wave front lying in $x_{1} \leqq 0$ has physical meaning. Let $F^{\prime}=\left(\eta^{\prime}, 0,0\right)$ be the focus of the hyperboloid, which is also the location of the original disturbance in the second medium. Let $P=\left(x_{1}, x_{2}, x_{3}\right)$ be a point of (23), i.e., a point of the apparent wave front at the time $t=0$. Let $Q$ be the intersection of the plane $x_{1}=0$ with the normal to the hyperboloid at $P$. One easily verifies that

$$
(1 / c) \overline{Q P}=\left(1 / c^{\prime}\right) \overline{Q F^{\prime}},
$$

and that the sines of the angles the lines $Q P$ and $Q F^{\prime}$ form with the $x_{1}$-axis are in the ratio $c / c^{\prime}$. That means that the points of the wave front at the time $t$ with $x_{1}<0$ can also be obtained by moving from $F^{\prime}$ with velocity $c^{\prime}$ along a line leading to a point $Q$ of $x_{1}=0$ and then proceeding with velocity $c$ along the direction obtained from Snell's law of refraction. (See Figure 1.)

The points on the plane $x_{1}=0$ in which $E, H$ can be different from 0 lie in or on the sphere of radius $c^{\prime} t$ about the point $F^{\prime}=\left(\eta^{\prime}, 0,0\right)$, i.e., lie in the set

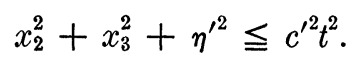

The vectors $E^{\prime}, H^{\prime}$ for $x_{1}^{\prime}=0$ have their support in the same set by virtue of the transition conditions. We can consider $E^{\prime}, H^{\prime}$ as solutions of the hyperbolic equations

$$
L^{\prime} E^{\prime}=L^{\prime} H^{\prime}=0 \quad \text { for } x_{1}^{\prime} \geqq 0,
$$




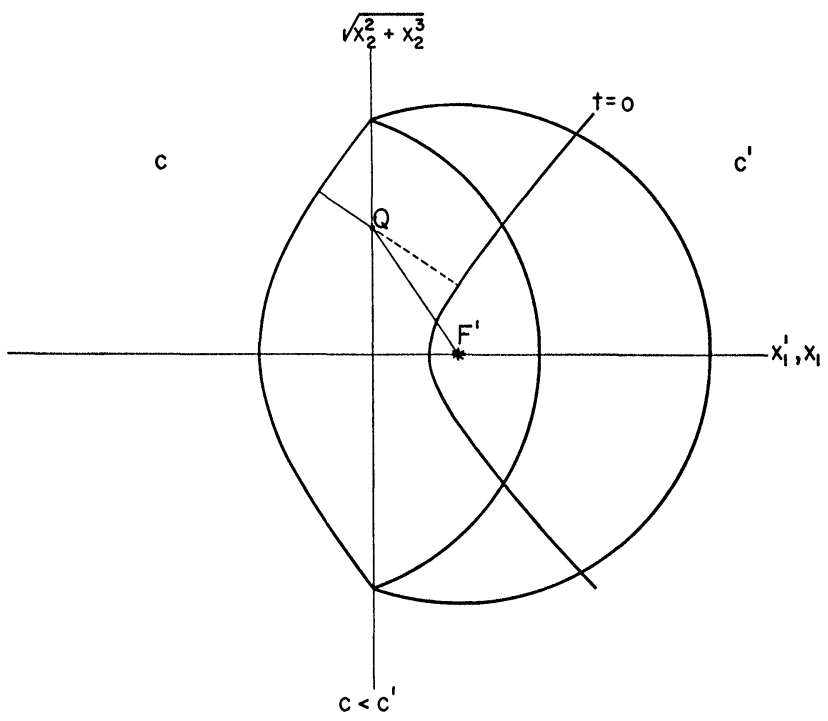

Figure 1

which have their initial values for $t=0, x_{1}^{\prime} \geqq 0$ concentrated at $F^{\prime \prime}$, and have nonvanishing boundary data on $x_{1}^{\prime}=0$ only in the portion (24) of the boundary plane. The contribution of the initial disturbance to the state of $E^{\prime}, H^{\prime}$ at the time $t$ is confined to the sphere

$$
\left(x_{1}^{\prime}-\eta^{\prime}\right)^{2}+x_{2}^{2}+x_{3}^{2}={c^{\prime}}^{2} t^{2} .
$$

One easily verifies that the contribution of the boundary data is confined to the symmetric sphere

$$
\left(x_{1}^{\prime}+\eta^{\prime}\right)^{2}+x_{2}^{2}+x_{3}^{2} \leqq c^{\prime 2} t^{2} .
$$

Hence $E^{\prime}, H^{\prime}$ have their support at the time $t$ in the union of the sets (25a) and $(25 \mathrm{~b})$.

Next we consider the effect of an initial disturbance concentrated at a point $F=(\eta, 0,0)$ of the first medium:

$$
\eta<0 \text {. }
$$

The vectors $e^{0}, h^{0}$ are solutions of the boundary-initial value problem (13a, $\mathrm{b}, \mathrm{c})$ for $x_{1} \leqq 0, x_{1}^{\prime} \geqq 0$. The initial data vanish, and the boundary data are concentrated at the point $y=\left(y_{1}, y_{1}^{\prime}, y_{2}, y_{3}\right)=(\eta, 0,0,0)$. Formula (15b) shows that $e^{0}, h^{0}$ will have their support in the set $R(y-x)=0$, i.e., on

$$
\left(x_{1}-\eta\right)-\frac{1}{p q} x_{1}^{\prime 2}-\frac{1}{p q-1}\left(x_{2}^{2}+x_{3}^{2}\right)=0, \quad x_{1} \leqq \eta, \quad x_{1}^{\prime} \geqq 0 .
$$


The same holds for $u^{0}, v^{0}, \phi^{0}, \psi^{0}$. Those quantities continued as odd functions of $x_{1}$ will then have their support for $x_{1} \geqq 0, x_{1}^{\prime} \geqq 0$ in the symmetric set

$$
\left(x_{1}+\eta\right)^{2}-\frac{1}{p q} x_{1}^{\prime 2}-\frac{1}{p q-1}\left(x_{2}^{2}+x_{3}^{2}\right)=0, \quad x_{1} \geqq-\eta, \quad x_{1}^{\prime} \geqq 0 .
$$

The vectors $e^{0}, h^{0}$ for $x_{1} \geqq 0, x_{1}^{\prime} \geqq 0$ are obtained by integrating along lines

$$
p d x_{1}+d x_{1}^{\prime}=d x_{2}=d x_{3}=0
$$

or

$$
q d x_{1}+d x_{1}^{\prime}=d x_{2}=d x_{3}=0 .
$$

It follows that the support of $e^{0}, h^{0}$ for $x_{1} \geqq 0, x_{1}^{\prime} \geqq 0$ is confined to the set

$$
\left(x_{1}+\eta\right)^{2}-\frac{1}{p q} x_{1}^{\prime 2}-\frac{1}{p q-1}\left(x_{2}^{2}+x_{3}^{2}\right) \geqq 0, \quad x_{1} \geqq-\eta, \quad x_{1}^{\prime} \geqq 0 .
$$

For $x_{1}^{\prime}=0$ the vectors $e^{0}, h^{0}$ reduce to $E^{0}, H^{0}$. It follows that the field $E, H$ can be obtained by solving a pure initial value problem for $E, H$ as solutions of

$$
L E=L H=0
$$

with initial values for $t=0$ confined to the point

and to the conical set

$$
F=(\eta, 0,0)
$$

$$
\left(x_{1}+\eta\right)^{2}-\frac{1}{p q-1}\left(x_{2}^{2}+x_{3}^{2}\right) \geqq 0, \quad x_{1} \geqq-\eta .
$$

It follows that at the time $t$ the field $E, H$ has its support in the union of the sphere

$$
\left(x_{1}-\eta\right)^{2}+x_{2}^{2}+x_{3}^{2}=c^{2} t^{2}
$$

and of the $c t$-neighborhood of (28). The $c t$-neighborhood of the set (18) consists of the truncated cone

$\left(x_{1}+\eta+\frac{c t \sqrt{p q}}{\sqrt{p q-1}}\right)^{2}-\frac{1}{p q-1}\left(x_{2}^{2}+x_{3}^{2}\right) \geqq 0, \quad x_{1} \geqq-\eta-\frac{c t \sqrt{p q-1}}{\sqrt{p q}}$ and the spherical cap

$$
\left(x_{1}+\eta\right)^{2}+x_{2}^{2}+x_{3}^{2} \leqq c^{2} t^{2}, \quad-\eta-c t \leqq x_{1} \leqq-\eta-c t \frac{\sqrt{p q-1}}{\sqrt{p q}} .
$$

(See Figure 2.)

For the purpose of finding the support of the field $E^{\prime}, H^{\prime}$ we first find that of $e, h$ and then use that

$$
E^{\prime}=(P e)_{x_{1}=0}, \quad H^{\prime}=(Q h)_{x_{1}=0} .
$$

The vectors $e^{0}, h^{0}$ have their support in the union of the sets (26), (27). For 


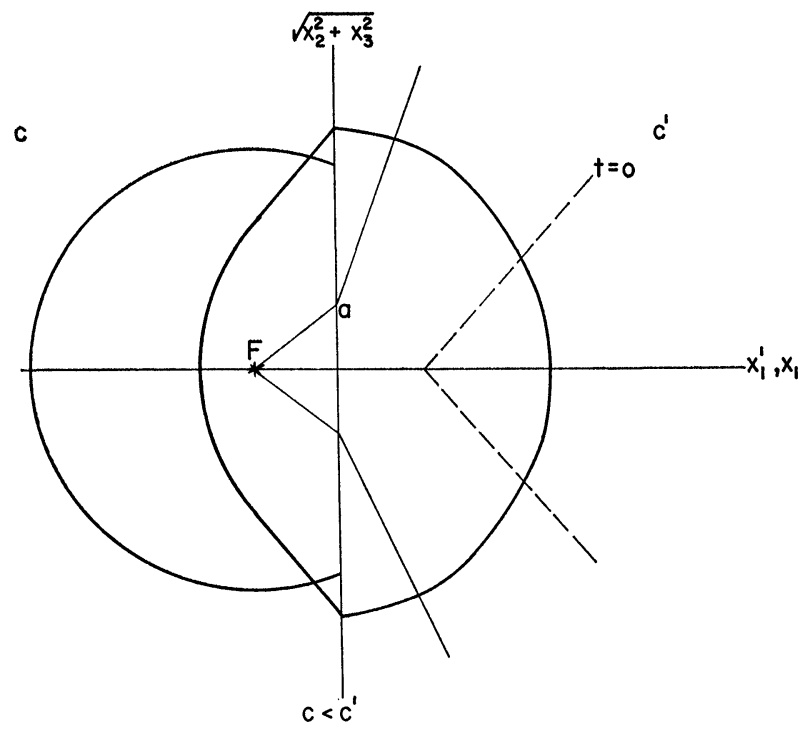

Figure 2

any fixed $x_{1}^{\prime} \geqq 0$ the vectors $e, h$ are the solutions of the pure initial value problem $(13 \mathrm{f}, \mathrm{g})$. It follows that for fixed $x_{1}^{\prime} \geqq 0$ and positive $t$ the support of $e, h$ is contained in the $c t$-neighborhood of the sets (26), (27) in $x_{1} x_{2} x_{3}$ space. The plane $x_{1}=0$ in $x_{1} x_{2} x_{3}$-space lies outside the sets (26), (27). A point of that plane lies in a $c t$-neighborhood of the sets $(26),(27)$ if it lies in a $c t$-neighborhood of a boundary point of one of the sets. Since the boundaries of the sets $(26),(27)$ are symmetric with respect to the plane $x_{1}=0$, we find that it is sufficient to consider the ct-neighborhood of the set (26). We see then that $E^{\prime}\left(x_{1}^{\prime}, x_{2}, x_{3}, t\right), H^{\prime}\left(x_{1}^{\prime}, x_{2}, x_{3}, t\right)$ can be different from 0 for $x_{1}^{\prime} \geqq 0, t>0$ only, if there exist $y_{1}, y_{2}, y_{3}$ with

$$
\begin{gathered}
\left(y_{1}-\eta\right)^{2}-\frac{1}{p q} x_{1}^{\prime 2}-\frac{1}{p q-1}\left(y_{2}^{2}+y_{3}^{2}\right)=0, \quad y_{1} \leqq \eta, \\
y_{1}^{2}+\left(x_{2}-y_{2}\right)^{2}+\left(x_{3}-y_{3}\right)^{2} \leqq c^{2} t^{2} .
\end{gathered}
$$

We can take here for $y_{1}, y_{2}, y_{3}$ those quantities satisfying (29), for which the left-hand side of (30) assumes its smallest value. Then

are proportional to

$$
y_{1}, \quad x_{2}-y_{2}, \quad x_{3}-y_{3}
$$

Introducing

$$
y_{1}-\eta, \quad \frac{1}{p q-1} y_{2}, \quad \frac{1}{p q-1} y_{3}
$$

$$
\left(z_{1}, z_{2}, z_{3}\right)=\left(\eta, \frac{p q}{p q-1} y_{2}, \frac{p q}{p q-1} y_{3}\right)
$$


we also have

$$
\left(z_{1}, z_{2}, z_{3}\right)=\left(\theta y_{1}, \theta y_{2}+(1-\theta) x_{2}, \theta y_{3}+(1-\theta) x_{3}\right)
$$

where $\theta=\eta / y_{1}$ lies between 0 and 1. By (29), (31)

$$
\begin{aligned}
\sqrt{p q} \sqrt{\left(y_{1}-z_{1}\right)^{2}+\left(y_{2}-z_{2}\right)^{2}+\left(y_{3}-z_{3}\right)^{2}} & \\
& =\sqrt{\left(z_{1}-\eta+x_{1}^{\prime}\right)^{2}+z_{2}^{2}+z_{3}^{2}},
\end{aligned}
$$

while by (32)

$$
\begin{aligned}
& \sqrt{y_{1}^{2}+\left(y_{2}-x_{2}\right)^{2}+\left(y_{3}-x_{3}\right)^{2}} \\
& =\sqrt{\left(y_{1}-z_{1}\right)^{2}+\left(y_{2}-z_{2}\right)^{2}+\left(y_{3}-z_{3}\right)^{2}} \\
& \quad+\sqrt{z_{1}^{2}+\left(z_{2}-x_{2}\right)^{2}+\left(z_{3}-x_{3}\right)^{2}} .
\end{aligned}
$$

It follows from (33), (34), (30) that

$$
c^{\prime-1} \sqrt{\left(z_{1}-\eta+x_{1}^{\prime}\right)^{2}+z_{2}^{2}+z_{3}^{2}}+c^{-1} \sqrt{z_{1}^{2}+\left(z_{2}-x_{2}\right)^{2}+\left(z_{3}-x_{3}\right)^{2}} \leqq t .
$$

Introducing finally $a_{1}, a_{2}, a_{3}$ by

$$
a_{1}=0, \quad a_{2}=x_{2}-z_{2}, \quad a_{3}=x_{3}-z_{3}
$$

we see that in order that $E^{\prime}, H^{\prime}$ do not vanish at the point $\left(x_{1}^{\prime}, x_{2}, x_{3}\right)$ it is necessary that there exist quantities $a_{2}, a_{3}$ such that

$$
c^{-1} \sqrt{\eta^{2}+a_{2}^{2}+a_{3}^{2}}+c^{\prime-1} \sqrt{x_{1}^{\prime 2}+\left(x_{2}-a_{2}\right)^{2}+\left(x_{3}-a_{3}\right)^{2}} \leqq t .
$$

Relation (35) expresses that there exists a point $\left(0, a_{2}, a_{3}\right)$ such that moving from $(\eta, 0,0)$ to $\left(0, a_{2}, a_{3}\right)$ with speed $c$ and then from $\left(0, a_{2}, a_{3}\right)$ to $\left(x_{1}^{\prime}, x_{2}, x_{3}\right)$ with speed $c^{\prime}$ will take a total time at most $t$. That is, the support of $E^{\prime}, H^{\prime}$ due to an initial disturbance at $(\eta, 0,0)$ is contained in the set given by the law of refraction of geometrical optics.

\section{REFERENCES}

1. E. Geruoy, Total reflection of waves from a point source, Comm. Pure Appl. Math., vol. 6 (1953), pp. 73-91.

2. H. G. Garnir, Propagation de l'onde émise par une source ponctuelle et instantanée dans un dioptre plan, Bull. Soc. Roy. Sci. Liège, vol. 22 (1953), pp. 85-100, 148-162.

3. F. Jown, Solutions of second order hyperbolic differential equations with constant coefficients in a domain with a plane boundary, Comm. Pure Appl. Math., vol. 7 (1954), pp. 245-269.

4. - - On linear partial differential equations with analytic coefficients, Comm. Pure Appl. Math., vol. 2 (1949), pp. 209-253.

5. E. HolmgReN, Ueber Systeme von linearen partiellen Differentialgleichungen, Öf versigt af Kongl. Vetenskaps-Akademiens Förhandlingar (Stockholm), vol. 58 (1901), pp. 91-103.

6. H. FREDA, Méthode des caractéristiques pour l'intégration des équations aux dérivées partielles linéaires hyperboliques, Mémorial des Sciences Mathématiques, fasc. 84, Paris, Gauthier-Villars, 1937. 
7. H. Poritsky, Propagation of transient fields from dipoles near the ground, British Journal of Applied Physics, vol. 6 (1955), pp. 421-426.

8. A. Sommerfeld Über die Ausbreitung der Wellen in der drahtlosen Telegraphie, Ann. Physik (4), vol. 28 (1909), pp. 665-736 and vol. 81 (1926), pp. 1135-1153.

9. H. WEYL, Ausbreitungen elektromagnetischer Wellen über einem ebenen Leiter, Ann. Physik (4), vol. 60 (1919), pp. 481-500.

10. B. VAN DER PoL, On discontinuous electromagnetic waves and the occurrence of a surface wave, Transactions of the Institute of Radio Engineers, Professional Group on Antennas and Propagation, vol. 4 (1956), pp. 288-293.

\section{New York University}

NEw York, NEw YORK 